\title{
Anisotropic Complementary Acoustic Metamaterial for Canceling out Aberrating Layers
}

\author{
Chen Shen, ${ }^{1}$ Jun $\mathrm{Xu},{ }^{2, *,+}$ Nicholas X. Fang, ${ }^{2}$ and Yun Jing ${ }^{1, *, \dagger}$ \\ ${ }^{1}$ Department of Mechanical and Aerospace Engineering, North Carolina State University, \\ Raleigh, North Carolina 27695, USA \\ ${ }^{2}$ Department of Mechanical Engineering, Massachusetts Institute of Technology, \\ Cambridge, Massachusetts 02139, USA \\ (Received 14 August 2014; published 19 November 2014)
}

\begin{abstract}
In this paper, we investigate a type of anisotropic, acoustic complementary metamaterial (CMM) and its application in restoring acoustic fields distorted by aberrating layers. The proposed quasi two-dimensional (2D), nonresonant CMM consists of unit cells formed by membranes and side branches with open ends. Simultaneously, anisotropic and negative density is achieved by assigning membranes facing each direction ( $x$ and $y$ directions) different thicknesses, while the compressibility is tuned by the side branches. Numerical examples demonstrate that the CMM, when placed adjacent to a strongly aberrating layer, could acoustically cancel out that aberrating layer. This leads to dramatically reduced acoustic field distortion and enhanced sound transmission, therefore virtually removing the layer in a noninvasive manner. In the example where a focused beam is studied, using the CMM, the acoustic intensity at the focus is increased from $28 \%$ to $88 \%$ of the intensity in the control case (in the absence of the aberrating layer and the CMM). The proposed acoustic CMM has a wide realm of potential applications, such as cloaking, all-angle antireflection layers, ultrasound imaging, detection, and treatment through aberrating layers.
\end{abstract}

DOI: 10.1103/PhysRevX.4.041033

In many medical ultrasound or nondestructive evaluation (NDE) applications, ultrasound needs to be transmitted through an aberrating layer [1-7], where either the transmission is desired to be maximized or the reflection needs to be minimized. One of the most representative examples is transcranial ultrasound beam focusing, which could find usage in both brain imaging and treatment $[6,7]$. However, transcranial beam focusing is extremely challenging because of the presence of the skull. A common approach to achieve transcranial beam focusing is based on the timereversal or phase-conjugate technique and ultrasound phased arrays $[8,9]$. Although the focal position can be corrected, one significant shortcoming of this strategy is that it does not compensate for the large acoustic energy loss due to the impedance mismatch between the skull and the background medium (water). Recent development of acoustic metamaterials [10-12] could open up the possibility for noninvasive ultrasound transmission through aberrating layers. For example, an acoustic metamaterial could be used to cancel out or cloak the aberrating layer, allowing the acoustic wave to pass through the layer without energy loss (Fig. 1). Conventional cloaking

\footnotetext{
${ }^{*}$ To whom all correspondence should be addressed. †yjing2@ncsu.edu

*xujun@mit.edu
}

Published by the American Physical Society under the terms of the Creative Commons Attribution 3.0 License. Further distribution of this work must maintain attribution to the author(s) and the published article's title, journal citation, and DOI.
Subject Areas: Acoustics, Metamaterials

strategies $[11,13,14]$, however, compress the space and hide the object inside an enclosure in which there is no interaction with the outside world; therefore, it is not suited to the problem of interest in this study. Lai et al. demonstrated that cloaking or illusion based on electromagnetic wave (EM) complementary metamaterials (CMM) [15] can open up a virtual hole in a wall without distortion [16,17]. In addition, this type of approach does not require the cloaked object to be inside an enclosure or cloaking shell, and it is valid in free space [18]. Because of the similarity between acoustic and EM wave equations in two dimensions (2D), CMMs have also been proposed for acoustic cloaking $[19,20]$. The simulations, however, were based on homogenized effective media, and no explicit design was given.

In this paper, we aim to fill the long-standing gap between the theory of CMM and its design in the context of acoustics and to further apply it to ultrasound transmission through aberrating layers. A generalized CMM requires negative and anisotropic properties simultaneously, and preferably should not be resonance based because of the large resonance damping. Such a CMM in acoustics has not been achieved, to the best of our knowledge. We present here a design of a quasi-2D acoustic CMM utilizing clamped membranes and side branches. By assigning membranes in different directions with different thicknesses, the effective density is shown to be both anisotropic and negative at the operating frequency. As shown in this study, the proposed CMMs can effectively cancel out aberrating layers in order to allow the sound to 


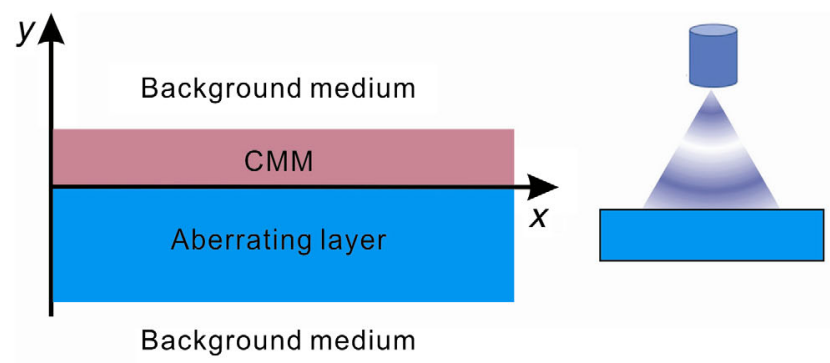

(a)

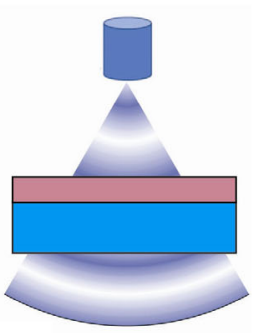

(b)

FIG. 1. (a) Schematic of the CMM, the aberrating layer, and the background medium. (b) Concept of the CMM for enhancing the sound transmission. The acoustic energy is blocked by the aberrating layer due to the large impedance mismatch (left), while it can transmit with high efficiency if placing the desired CMM adjacent to the aberrating layer (right).

pass through with strongly enhanced transmission and reduced acoustic field distortion.

The CMM is placed on top of the aberrating layer, as illustrated in Fig. 1(a). The aberrating layer is assumed to be sufficiently long so that the edges do not significantly affect the acoustic field. The CMM compresses and cancels the information of the selected aberrating layer. Let $\rho^{(c)}\left(x^{(c)}, y^{(c)}, z^{(c)}\right), \quad \beta^{(c)}\left(x^{(c)}, y^{(c)}, z^{(c)}\right)$ and $\rho^{(a)}\left(x^{(a)}, y^{(a)}, z^{(a)}\right), \quad \beta^{(a)}\left(x^{(a)}, y^{(a)}, z^{(a)}\right)$ be the effective density and compressibility tensors of the CMM and the aberrating layer, respectively. $x^{(c)}, y^{(c)}, z^{(c)}$ and $x^{(a)}, y^{(a)}$, $z^{(a)}$ are generalized curved coordinates. Based on the acoustic coordinate transformation, we have [10]

$$
\begin{gathered}
{\left[\rho^{(c)}\right]^{-1}=\mathbf{A}\left[\rho^{(a)}\right]^{-1} \mathbf{A}^{T} / \operatorname{det} \mathbf{A},} \\
\beta^{(c)}=\beta^{(a)} / \operatorname{det} \mathbf{A},
\end{gathered}
$$

where $\mathbf{A}$ is the Jacobian transformation tensor of compressing transformation given by

$$
\mathbf{A}=\left[\begin{array}{lll}
\frac{\partial x^{(c)}}{\partial x^{(a)}} & \frac{\partial x^{(c)}}{\partial y^{(a)}} & \frac{\partial x^{(c)}}{\partial z^{(a)}} \\
\frac{\partial y^{(c)}}{\partial x^{(a)}} & \frac{\partial y^{(c)}}{\partial y^{(a)}} & \frac{\partial y^{(c)}}{\partial z^{(a)}} \\
\frac{\partial z^{(c)}}{\partial x^{(a)}} & \frac{\partial z^{(c)}}{\partial y^{(a)}} & \frac{\partial z^{(c)}}{\partial z^{(a)}}
\end{array}\right] .
$$

This study focuses on 2D problems, and only wave propagation in the $x-y$ plane is of interest. The $z$ component in the Jacobian matrix is therefore dropped. Without loss of generality, the thickness of the CMM is assumed to be half of the aberrating layer, which leads to

$$
\frac{\partial x^{(c)}}{\partial x^{(a)}}=1
$$

because of the coordinate transformation $x^{(c)}=x^{(a)}$. The following equation needs to be satisfied because of the coordinate transformation $y^{(c)}=-y^{(a)}$, such that

$$
\frac{\partial y^{(c)}}{\partial y^{(a)}}=-0.5
$$

This ratio is negative since the acoustic information is folded in the CMM and would cancel out that of the aberrating layer. Other components, $\partial x^{(c)} / \partial y^{(a)}$ and $\partial y^{(c)} / \partial x^{(a)}$, in the tensor are equal to zero, as the transformation in each direction (i.e., $x$ and $y$ directions) are independent. For more complicated geometries, offdiagonal components may appear. They could, however, be eliminated by coordinate rotations [21].

Finally, tensor $\mathbf{A}$ reads

$$
\mathbf{A}=\left[\begin{array}{cc}
1 & 0 \\
0 & -0.5
\end{array}\right]
$$

Consequently, the density and compressibility tensors of the CMM are

$$
\begin{gathered}
\rho^{(c)}=\left[\begin{array}{cc}
-0.5 & 0 \\
0 & -2
\end{array}\right] \times \rho^{(a)}, \\
\beta^{(c)}=-2 \times \beta^{(a)} .
\end{gathered}
$$

Such a generalized CMM requires strongly anisotropic density as well as negativity for both density and compressibility. We note that the density can be isotropic if the thickness of the CMM is chosen to be the same as the aberrating layer. In this case, however, the refractive index is -1 , and the $k$ vector along the interface goes to infinity [22]. In other words, such a CMM will be very unit-cellsize sensitive and can be difficult to demonstrate. Furthermore, a generalized CMM is preferred in practice as its thickness can be arbitrarily chosen; i.e., it does not depend on the thickness of the aberrating layer, thus providing great flexibility. As shown below, the proposed CMMs can be achieved by periodic cubic blocks with clamped elastic membranes and side branches, and they are nonresonant acoustic metamaterials.

A portion of the CMM ( $5 \times 5$ units $)$ is shown in Fig. 2(a). The membrane size is $2 \mathrm{~mm} \times 2 \mathrm{~mm}$, and the membrane 


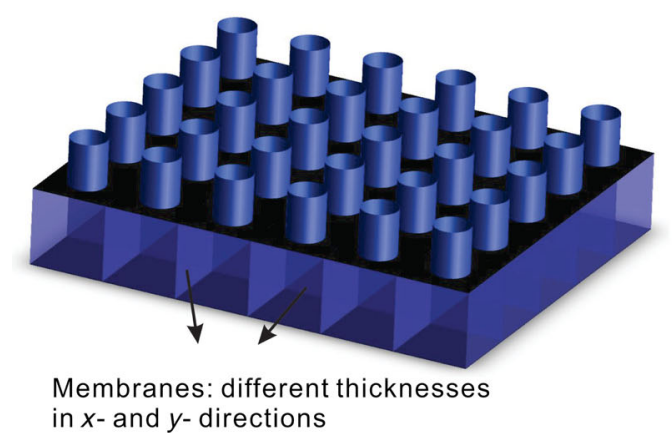

(a)

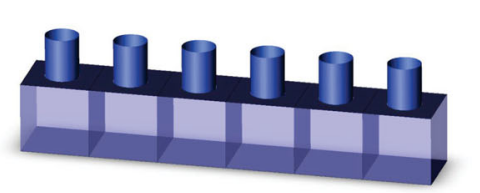

(b)

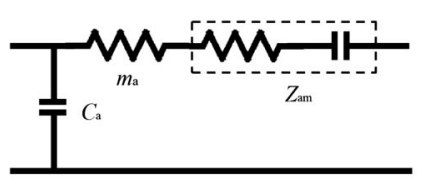

(c)

FIG. 2. (a) Schematic of a portion of the quasi-2D CMM. (b) Schematic of a 1D side branch and membrane-based metamaterial. (c) Equivalent acoustic circuit of the 1D membrane-based metamaterial.

material is aluminum; the Young modulus is $70 \mathrm{GPa}$; the Poisson ratio is 0.33 ; the density is $2700 \mathrm{~kg} / \mathrm{m}^{3}$; the tension on the membrane is assumed to be zero. The membrane is introduced here to tune the density. The side branches are open ended and are introduced to tune the compressibility. The operating frequency of the CMM is $50 \mathrm{kHz}$, at which the wavelength in water (background medium) is 15 times larger than the size of a unit cell. By adjusting the thicknesses of the membranes facing each direction ( $x$ and $y$ ), the effective density can be tuned therein in order to achieve anisotropy. Assuming the interaction and coupling between membranes in the $x$ and $y$ directions is negligible, the effective density and compressibility in either the $x$ or $y$ direction can be estimated separately by one-dimensional (1D) studies [23]. To this end, 1D models are first studied in order to determine the appropriate thicknesses of the membranes and the dimensions of the side branches by both theoretical analysis and numerical simulations [Fig. 2(b)]. Two separate sets of simulations are conducted for the $x$ and $y$ directions, respectively. The finite-element analysis software COMSOL $4.3 \mathrm{~b}$ is used for all simulations. The effective density and compressibility of the proposed CMM can be extracted from numerical-simulation results by a finitedifference approximation method [24]. On the other hand, the effective compressibility with open-ended side branches, in theory, can be written as $[25,26]$

$$
\beta_{e}=\beta_{0}\left(1-\frac{S}{A d \rho_{0} \beta_{0} l_{e} \omega^{2}}\right)
$$

where $\beta_{e}$ is the effective compressibility, $\beta_{0}$ is the background-medium compressibility, $S$ is the cross-section area of the branch, $A$ is the cross-section area of the waveguide (cubic block), $d$ is the length of the unit cell, $l_{e}$ is the effective length of the branch, and $\omega$ is the angular frequency.

It has been demonstrated that for the 1D membranebased metamaterial shown in Fig. 2(b), the effective density depends on the properties of the membranes [25,27-29]. The side branches are assumed to have a negligible effect on the effective density [25]. The effective density with clamped membranes can be derived by using the lumped model. Figure 2(c) shows the equivalent acoustic circuit of the 1D membrane-based metamaterial, where $m_{a}$ is the effective acoustic mass of the tube, $C_{a}$ is the acoustic capacitance of the waveguide, and $Z_{a m}$ is the acoustic impedance of the membrane, which can be approximated by an inductor and capacitor in series in the low-frequency region [27].

$m_{a}$ and $C_{a}$ are given by [27]

$$
\begin{gathered}
m_{a}=\frac{\rho_{0}}{A(d-h),} \\
C_{a}=A \beta_{0}(d-h),
\end{gathered}
$$

where $\rho_{0}$ is the background-medium density and $h$ is the membrane thickness.

The acoustic impedance of the membrane, $Z_{a m}$, can be defined as [30]

$$
Z_{a m}=\frac{Z_{m}}{A^{2}}=\frac{\iint \Delta p d A}{j \omega \xi A^{2}},
$$

where $\Delta p=p_{1}-p_{2}$ is the pressure difference across the membrane, $Z_{m}$ is the mechanical impedance of the membrane, and $\xi$ represents the average displacement of the membrane. For circular membranes, an analytical solution exists for Eq. (12). In this study, Eq. (12) is evaluated by finite-element analysis since square membranes are used.

The combination of Eqs. (10) and (12) yields the expression for the total acoustic impedance of the tube:

$$
Z_{a s}=j \omega m_{a}+Z_{a m} .
$$

The effective density of a unit cell is then given by [27]

$$
\rho_{e}=\frac{Z_{a s}}{j \omega} \cdot \frac{1}{d A} .
$$

The thicknesses of the membranes are $0.083 \mathrm{~mm}$ and $0.11 \mathrm{~mm}$ in the $x$ and $y$ directions, respectively. 


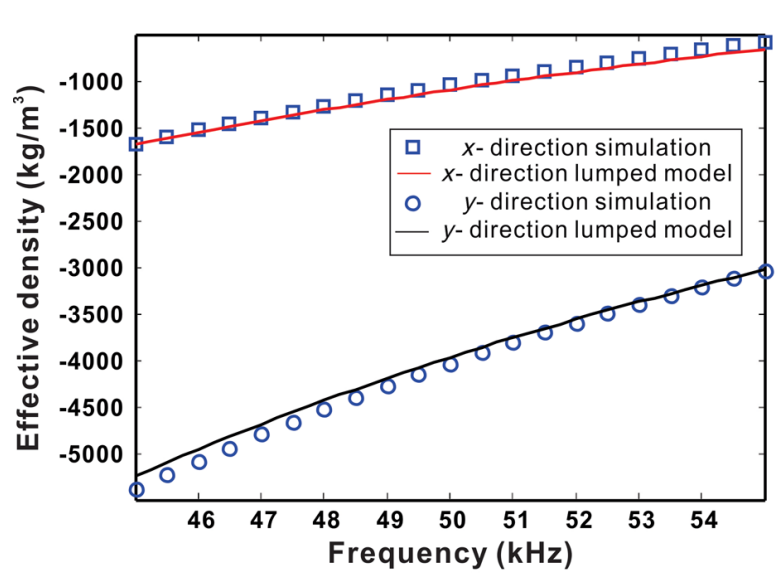

(a)

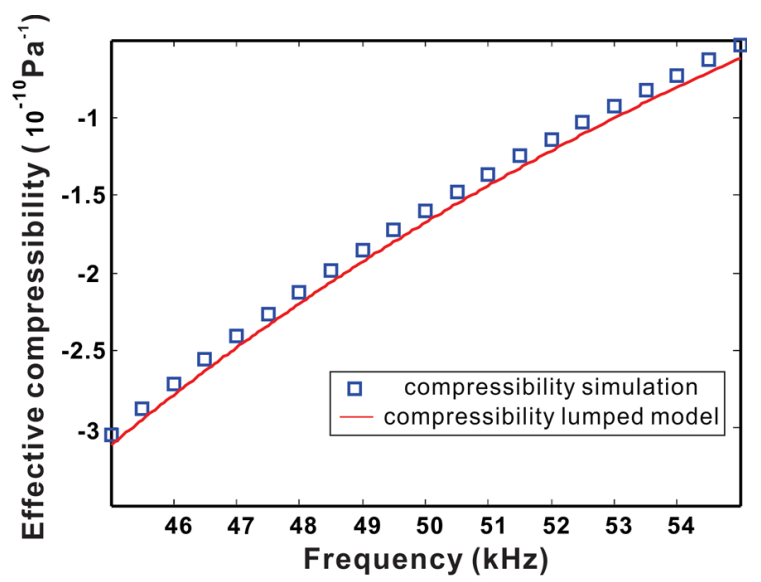

(b)

FIG. 3. Effective densities and compressibility extracted from full-wave simulations and predicted by the lumped model. (a) Effective densities in the $x$ and $y$ directions. (b) Effective compressibility. At $50 \mathrm{kHz}$, the desired density and compressibility are achieved.

The dimensions of the side branches are $r=0.5 \mathrm{~mm}$ and $h=1.25 \mathrm{~mm}$, respectively, where $r$ and $h$ are the radius and the height of the side branch, respectively. The effective acoustic parameters are compared with the theoretical prediction and are shown in Fig. 3, where good agreement can be observed. It is clear that these structures produce negative properties without relying on the resonance since the negative properties appear in a broadband frequency range.

Two sets of full-wave simulations are then carried out to validate the proposed CMM. The commercial package COMSOL MULTIPHYSICS $4.3 \mathrm{~b}$ is adopted throughout the modeling. The acoustic-shell interaction model is used for numerical simulations. Specifically, we use the interior shell feature to model the membranes. The entire CMM consists of $120 \times 10$ unit cells: there are 120 units in the $x$ direction and 10 units in the $y$ direction. Perfectly matched layers (PML) are used to minimize reflections from the boundary. The thickness of the aberrating layer is $40 \mathrm{~mm}$. The density and sound speed of the background medium (water) and the aberrating layer are $1000 \mathrm{~kg} / \mathrm{m}^{3}, 1500 \mathrm{~m} / \mathrm{s}$ and $2000 \mathrm{~kg} / \mathrm{m}^{3}, 2500 \mathrm{~m} / \mathrm{s}$, respectively. The properties of the aberrating layer are chosen so that it mimics human skulls. The acoustic impedance of the aberrating layer is therefore over 3.3 times larger than the background medium, providing a sufficient amount of mismatch. Figure 3 shows that at $50 \mathrm{kHz}$, the density and compressibility of CMM satisfy Eqs. (7) and (8) for the given materials: The density is $-1000 \mathrm{~kg} / \mathrm{m}^{3}$ and $-4000 \mathrm{~kg} / \mathrm{m}^{3}$ in the $x$ and $y$ directions, respectively; the compressibility is $-1.6 \times 10^{-10}$.

In the two examples, a curved (focused) array and a linear array are respectively introduced to generate different acoustic fields. In the first case, the curved array is placed in front of the aberrating layer (the CMM is on the same side as the array), whereas in the second case, the CMM is on the opposite side of the layer. The curved array is treated as an active acoustic source. Since the CMM would effectively cancel out the aberrating layer, the focal point will be $60 \mathrm{~mm}$ (total thickness of the CMM and the aberrating layer) behind the original one. The curved array is therefore moved $60 \mathrm{~mm}$ away from the aberrating layer when the $\mathrm{CMM}$ is considered, so the focal point remains at the desired location. The position of the linear array is not moved, as it is treated as a passive source. Figures 4(a)-4(f) show the acoustic intensity fields in three cases for each set of the simulation: with the skull but without CMM, homogeneous water (control case), and with CMM and the skull, respectively. When the CMM is used, the transmission acoustic energy is significantly enhanced compared to the one without the CMM, indicating that the reflected acoustic energy is also considerably reduced and the aberrating layer is acoustically canceled out. It can be observed in Fig. 4(c) that the transmission acoustic intensity field highly resembles that of the control case shown in Fig. 4(b), whereas in Fig. 4(f), the acoustic intensity field above the aberrating layer matches well with that of the control case shown in Fig. 4(e). Furthermore, as shown in Fig. 5, when the CMM and the skull are removed [edited out from Figs. 4(c) and 4(f)], the entire acoustic intensity fields look very similar to that of the control cases. In the curved array case, the intensity amplitude at the focal point is $88 \%$ of that of the control case. When CMM is not introduced, it is $28 \%$. This is an over $300 \%$ improvement in terms of the sound transmission. By varying the thickness of the aberrating layer, it is possible to have an even lower sound transmission (lower than 28\%); therefore, a more significant improvement is possible using CMMs. The CMM also results in an accurate focus position, which is only $3 \mathrm{~mm}(1 / 10$ of the wavelength) off the desired location, as in the control case. Without the CMM, the focus is $14 \mathrm{~mm}$ (about $1 / 2$ of the wavelength) off. This example demonstrates one potential application of CMMs to focus ultrasound behind aberrating layers, which could 


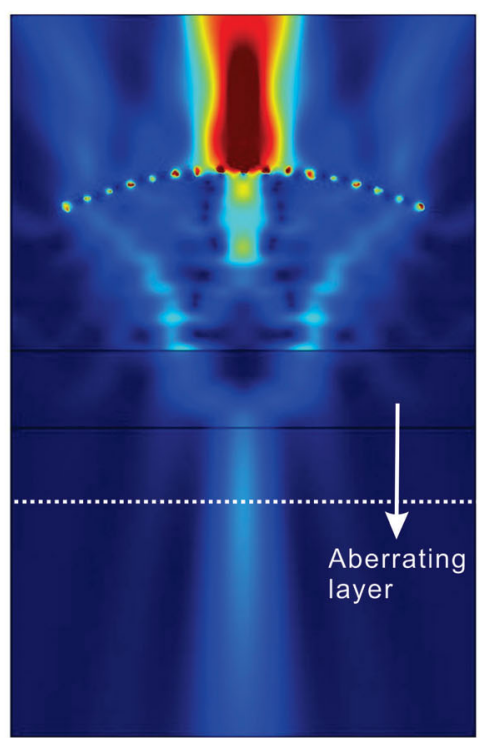

(a)

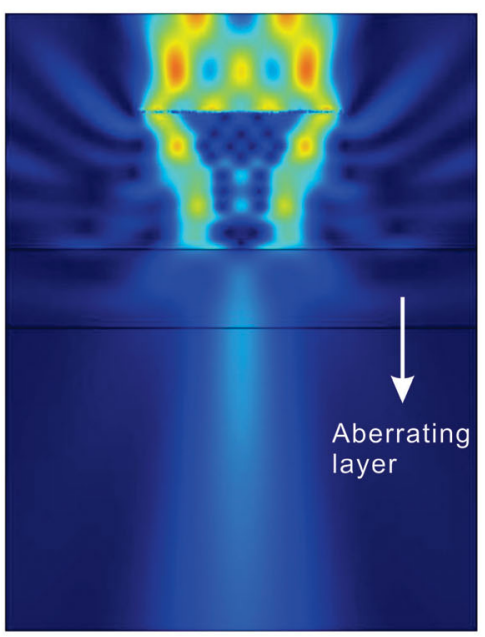

(d)

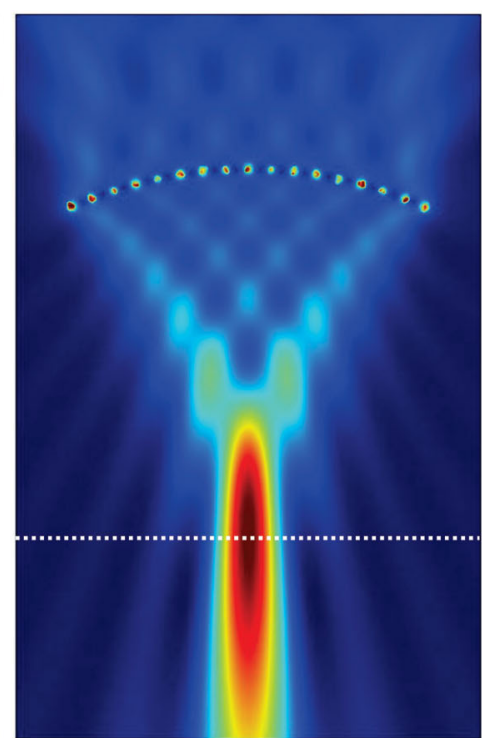

(b)

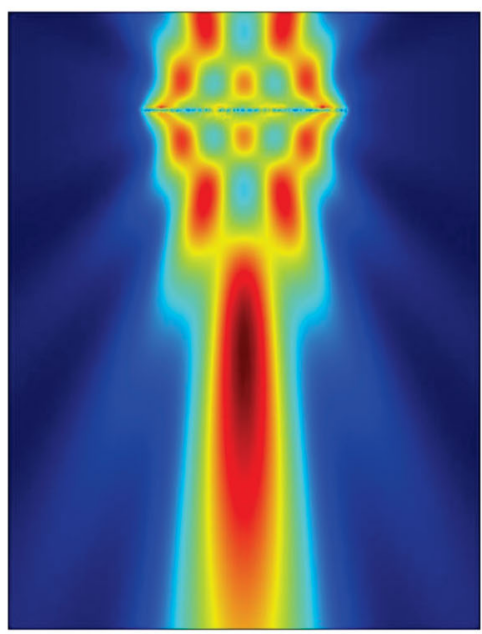

(e)

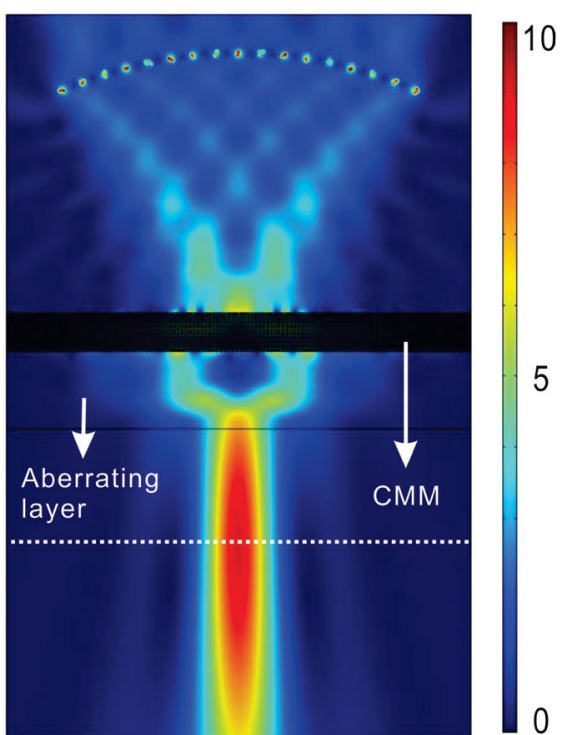

(c)

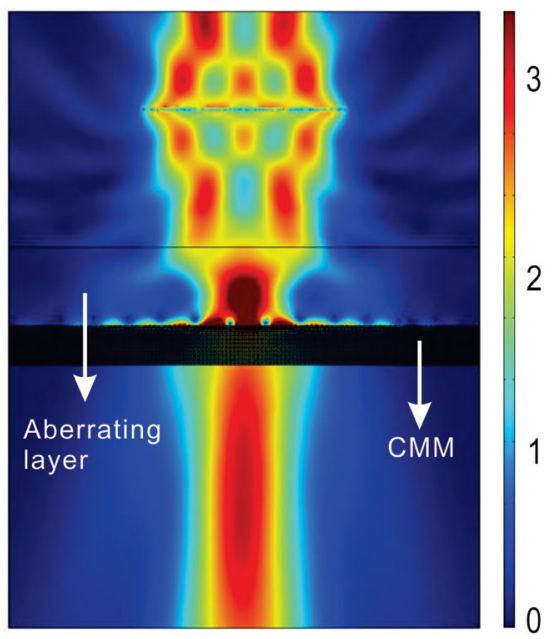

(f)

FIG. 4. Acoustic intensity field for a curved array (focused beam). The CMM is placed in front of the aberrating layer. White dotted lines indicate the position of the focal plane. Three cases are presented: (a) the skull only, (b) homogeneous medium, and (c) with CMM and the skull. Acoustic intensity field for a linear array (unfocused beam). The CMM is placed behind the aberrating layer. Three cases are presented: (d) skull only, (e) homogeneous medium, and (f) with CMM and the skull.

be extremely useful for improving ultrasound imaging or therapy. In the linear array case (unfocused acoustic field), the total acoustic intensity with the CMM behind the skull is $97 \%$ of that of the control case, whereas this parameter is $31 \%$ without the CMM. This example demonstrates another potential application of CMMs to detect passive acoustic source emissions or reflections from an object to be imaged or detected behind aberrating layers. Also very interestingly, this example indicates that the CMM can be placed behind a reflective layer and yet still achieve antireflection; thus, it has the advantage of being virtually concealed. Typical antireflection layers rely on sound absorption or damping and must be placed in front of the reflective layer; therefore, they could be aesthetically unpleasant, particularly when used for architectural acoustic applications. In both cases, the effective properties (density and compressibility) are extracted from the simulation results and are very close to the ones predicted by the earlier 1D model. Note that ideally a CMM should exactly restore the sound field without considering the energy loss inside the metamaterial. However, it would require an infinitely small unit cell so that the homogenization is perfect. The material losses are not taken into account in the simulations. However, aluminum (membrane material) is known to have a small loss factor, and the attenuation in water is also negligible. In addition, the side branch and membrane-based metamaterials used here are nonresonant metamaterials [25]; therefore, the material loss is not expected to be a significant factor. This is 


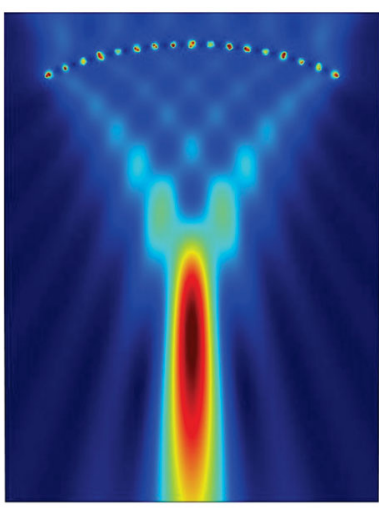

(a)

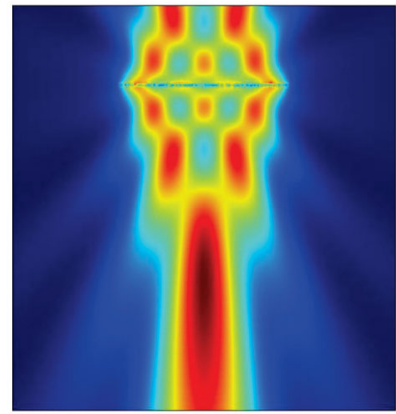

(c)

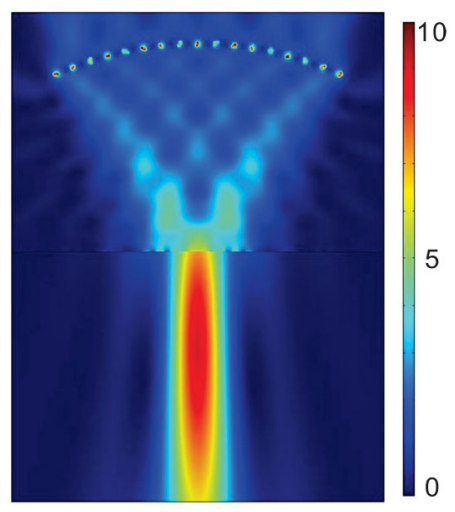

(b)

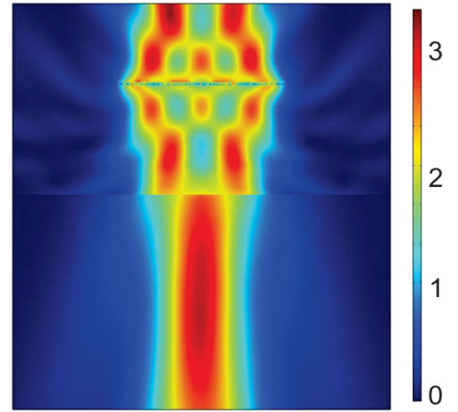

(d)
FIG. 5. (a) Focused beam, homogeneous medium. (b) Focused beam. The CMM and the skull are removed from Fig. 4(c), and the area above the CMM is moved downwards to be directly in touch with the area below the aberrating layer. (c) Unfocused beam, homogeneous medium. (d) Unfocused beam. The CMM and the skull are removed from Fig. 4(f), and the area above the aberrating layer is moved downwards to be directly in touch with the area below the CMM.

crucial for the application of interest in this paper, as our CMMs do not suffer from the energy loss due to the resonance which would inevitably occur in practice [31]. If the material losses are significant, the CMM can be designed so that its thickness is ultrathin in order to reduce the energy loss. Again, this is possible because the thickness of the CMM can be arbitrary in our design.

To conclude, the proposed acoustic CMM can be designed based on the coordinate transformation of acoustic waves and is able to cancel out an object in free space. Numerical examples are presented, in which CMMs are used to restore the acoustic fields distorted by aberrating layers. As demonstrated in the paper, the CMM is capable of virtually removing an aberrating layer in a noninvasive manner; therefore, it could greatly facilitate NDE, transcranial ultrasound imaging, and treatment. The proposed CMMs can be readily used to cancel out multiple layers [32] if needed. CMMs are also expected to be useful for the design of acoustic cloaking [32] and all-angle antireflection materials $[33,34]$. To extend the current design to $3 \mathrm{D}$, the branch openings can be replaced by slits, which also yield a negative compressibility or bulk modulus [27]. The major challenge in realizing the proposed CMM is that the thicknesses of the membranes should be reasonably accurate, as they determine the effective density of the metamaterial. The boundaries of the membranes should also be clamped as perfectly as possible. Finally, we note that, for a different piece of the skull, the acoustic properties (density and speed of sound) as well as the thickness could be different (variation around 10\%-20\%). This requires different effective density and compressibility for the CMM according to the coordinate transformation shown in the paper; otherwise, the CMM would not work effectively. Therefore, the same structure can be used, but the dimensions need to be modified (different membrane sizes, branch opening heights, etc.). This is, in some way, a theoretical limitation of CMMs; i.e., the properties of the CMM depend on the property or dimensions of the object to be canceled out. Nevertheless, the proposed design can be readily modified to achieve active CMMs; i.e., a single CMM can be tuned for canceling out different aberrating layers. For example, the aluminum membranes can be replaced by piezoelectric plates to actively tune the density [35].

Y. J. acknowledges the NC Space Grant New Investigator grant for financial support. J.X. and N. F. acknowledge the Multidisciplinary University Research Initiative from the Office of Naval Research for financial support through Grant No. N00014-13-1-0631.

[1] S. W. Smith, G. E. Trahey, and O. T. von Ramm, Phased Array Ultrasound Imaging through Planar Tissue Layers, Ultrasound Med. Biol. 12, 229 (1986).

[2] G. T. Clement, P. J. White, and K. Hynynen, Enhanced Ultrasound Transmission through the Human Skull Using Shear Mode Conversion, J. Acoust. Soc. Am. 115, 1356 (2004).

[3] N. M. Ivancevich, G. F. Pinton, H. A. Nicoletto, E. Bennett, D. T. Laskowitz, and S. W. Smith, Real-Time 3-D ContrastEnhanced Transcranial Ultrasound and Aberration Correction, Ultrasound Med. Biol. 34, 1387 (2008).

[4] K. K. Win, J. Wang, and C. Zhang, Identification and Removal of Reverberation in Ultrasound Imaging, 2010 5th IEEE Conf. Ind. Electron. Appl. (IEEE, Taichung, 2010), p. 1675.

[5] R. Demirli, M. G. Amin, X. Shen, and Y. D. Zhang, Ultrasonic Flaw Detection and Imaging through Reverberant Layers via Subspace Analysis and Projection, Adv. Acoust. Vib. 2012, 1 (2012).

[6] Y. Jing, F. C. Meral, and G. T. Clement, Time-Reversal Transcranial Ultrasound Beam Focusing Using a k-space Method, Phys. Med. Biol. 57, 901 (2012).

[7] T. Wang and Y. Jing, Transcranial Ultrasound Imaging with Speed of Sound-based Phase Correction: A Numerical Study, Phys. Med. Biol. 58, 6663 (2013).

[8] G. T. Clement and K. Hynynen, A Non-invasive Method for Focusing Ultrasound through the Human Skull, Phys. Med. Biol. 47, 1219 (2002). 
[9] M. Pernot, J.-F. Aubry, M. Tanter, A.-L. Boch, F. Marquet, M. Kujas, D. Seilhean, and M. Fink, In Vivo Transcranial Brain Surgery with an Ultrasonic Time Reversal Mirror, J. Neurosurg. 106, 1061 (2007).

[10] H. Chen and C. T. Chan, Acoustic Cloaking in Three Dimensions Using Acoustic Metamaterials, Appl. Phys. Lett. 91, 183518 (2007).

[11] S. Zhang, C. Xia, and N. Fang, Broadband Acoustic Cloak for Ultrasound Waves, Phys. Rev. Lett. 106, 024301 (2011).

[12] Z. Liang and J. Li, Extreme Acoustic Metamaterial by Coiling Up Space, Phys. Rev. Lett. 108, 114301 (2012).

[13] S. Cummer, B.-I. Popa, D. Schurig, D. Smith, J. Pendry, M. Rahm, and A. Starr, Scattering Theory Derivation of a 3D Acoustic Cloaking Shell, Phys. Rev. Lett. 100, 024301 (2008).

[14] M. Farhat, S. Enoch, S. Guenneau, and A. Movchan, Broadband Cylindrical Acoustic Cloak for Linear Surface Waves in a Fluid, Phys. Rev. Lett. 101, 134501 (2008).

[15] J. B. Pendry and S. A. Ramakrishna, Focusing Light Using Negative Refraction, J. Phys. Condens. Matter 15, 6345 (2003).

[16] Y. Lai, H. Chen, Z.-Q. Zhang, and C. Chan, Complementary Media Invisibility Cloak that Cloaks Objects at a Distance Outside the Cloaking Shell, Phys. Rev. Lett. 102, 093901 (2009).

[17] Y. Lai, J. Ng, H. Chen, D. Han, J. Xiao, Z.-Q. Zhang, and C. Chan, Illusion Optics: The Optical Transformation of an Object into Another Object, Phys. Rev. Lett. 102, 253902 (2009).

[18] X. Zhu, B. Liang, W. Kan, X. Zou, and J. Cheng, Acoustic Cloaking by a Superlens with Single-Negative Materials, Phys. Rev. Lett. 106, 014301 (2011).

[19] B. Liu and J. P. Huang, Acoustically Conceal an Object with Hearing, Eur. Phys. J. Appl. Phys. 48, 20501 (2009).

[20] J. Yang, M. Huang, C. Yang, J. Peng, and J. Chang, An External Acoustic Cloak with $N$-sided Regular Polygonal Cross Section Based on Complementary Medium, Comput. Mater. Sci. 49, 9 (2010).

[21] B.-I. Popa and S. A. Cummer, Homogeneous and Compact Acoustic Ground Cloaks, Phys. Rev. B 83, 224304 (2011).

[22] M. Ambati, N. Fang, C. Sun, and X. Zhang, Surface Resonant States and Superlensing in Acoustic Metamaterials, Phys. Rev. B 75, 195447 (2007).
[23] C. M. Park, J. J. Park, S. H. Lee, Y. M. Seo, C. K. Kim, and S. H. Lee, Amplification of Acoustic Evanescent Waves Using Metamaterial Slabs, Phys. Rev. Lett. 107, 194301 (2011).

[24] Y. Cheng, J. Xu, and X. Liu, One-Dimensional Structured Ultrasonic Metamaterials with Simultaneously Negative Dynamic Density and Modulus, Phys. Rev. B 77, 045134 (2008).

[25] S. H. Lee, C. M. Park, Y. M. Seo, Z. G. Wang, and C. K. Kim, Composite Acoustic Medium with Simultaneously Negative Density and Modulus, Phys. Rev. Lett. 104, 054301 (2010).

[26] C. Shen and Y. Jing, Side Branch-Based Acoustic Metamaterials with a Broad-Band Negative Bulk Modulus, Appl. Phys. A, doi:10.1007/s00339-014-8603-0 (2014).

[27] F. Bongard, H. Lissek, and J. R. Mosig, Acoustic Transmission Line Metamaterial with Negative/Zero/Positive Refractive Index, Phys. Rev. B 82, 094306 (2010).

[28] S. H. Lee, C. M. Park, Y. M. Seo, Z. G. Wang, and C. K. Kim, Acoustic Metamaterial with Negative Density, Phys. Lett. A 373, 4464 (2009).

[29] Y. Jing, J. Xu, and N. X. Fang, Numerical Study of a Near-Zero-Index Acoustic Metamaterial, Phys. Lett. A 376, 2834 (2012).

[30] Z. Skvor, Vibrating Systems and Their Equivalent Circuits (Elsevier, NewYork, 1991).

[31] N. Fang, D. Xi, J. Xu, M. Ambati, W. Srituravanich, C. Sun, and X. Zhang, Ultrasonic Metamaterials with Negative Modulus, Nat. Mater. 5, 452 (2006).

[32] See Supplemental Material at http://link.aps.org/ supplemental/10.1103/PhysRevX.4.041033 for the derivation and simulations for CMMs used for canceling out multiple aberrating layers and acoustic cloaking applications.

[33] D. Torrent and J. Sánchez-Dehesa, Acoustic Metamaterials for New Two-Dimensional Sonic Devices, New J. Phys. 9, 323 (2007).

[34] Y. Wang, K. Deng, S. Xu, C. Qiu, H. Yang, and Z. Liu, Applications of Antireflection Coatings in Sonic Crystalbased Acoustic Devices, Phys. Lett. A 375, 1348 (2011).

[35] W. Akl and A. Baz, Active Acoustic Metamaterial with Simultaneously Programmable Density and Bulk Modulus, J. Vib. Acoust. 135, 031001 (2013). 